\title{
La educación en energías renovables desde las controversias socio-científicas en la educación en Ciencias
}

\author{
Education in renewable energies from socio-scientific controversies in Science \\ Educação em energias renováveis de controvérias socio-científicas na educação em \\ Ciências
}

Vladimir Ballesteros-Ballesteros ${ }^{1}$
Adriana Patricia Gallego-Torres ${ }^{2}$

Recibido: febrero de 2019

Aceptado: abril de 2019

Para citar este artículo: Ballesteros-Ballesteros, V.; Gallego-Torres, A.P. (2019). La educación en energías renovables desde las controversias socio-científicas en la educación en Ciencias. Revista Científica, 35(2), 192-200. Doi: https:// doi.org/10.14483/23448350.14869

\section{Resumen}

El trabajo que aquí presentamos propone una reflexión teórica e histórica de las controversias socio-científicas. Estas se entienden acá como situaciones problematizadoras que se han convertido en una herramienta eficaz en la enseñanza de las ciencias, ya que permiten el desarrollo de procesos argumentativos, del pensamiento crítico, de concepciones más acordes a la ciencia y la actividad científica, al tiempo que alude a la historia y la filosofía. En este sentido, los lectores encontraran un breve recuento histórico del origen de las controversias socio-científicas y una propuesta para trabajar la educación en energías renovables.

Palabras clave: controversias socio-científicas, CTS, educación científica, educación en energías renovables.

\begin{abstract}
The work presented here proposes a theoretical and historical reflection of the socio-scientific controversies posed as problematizing situations that have become an effective tool in the teaching of sciences, since they allow the development of argumentative processes, the development of thought critical, conceptions more in line with science and scientific activity and allows use of history and philosophy. In this sense, readers will find a brief historical account of the origin of socio-scientific controversies and a proposal to work on Education in Renewable Energy.
\end{abstract}

Keywords: socio-scientific controversies, cts, scientific education, education in renewable energies. 


\section{Resumo}

O trabalho aqui apresentado propõe uma reflexão teórica e histórica das controvérsias sócio-científicas colocadas como situações problematizadoras que se tornaram uma ferramenta eficaz no ensino de ciências, pois permitem o desenvolvimento de processos argumentativos, o desenvolvimento do pensamento críticas, concepções mais em consonância com a ciência e atividade científica e permite o uso da história e da filosofia. Nesse sentido, os leitores encontrarão um breve relato histórico da origem das controvérsias sociocientíficas e uma proposta de trabalho em Educação em Energias Renováveis.

Palavras-chaves: controvérsias sociocientíficas, cts, educação científica, educação em energias renováveis.

\section{Introducción}

Uno de los primeros obstáculos de la ciencia y sus desarrollos se dio durante el siglo XVI, época conocida como "El oscurantismo" en la que la producción y difusión del conocimiento estaba restringida por motivos sociales, políticos y religiosos. Es decir, los conocimientos se limitaron a grupos selectos que podrían comprenderlos, razón por la cual se dijo que deberían ser escritos en lenguajes "oscuros" para que el público en general no pudiera comprenderlos. Este hecho puede traspolarse al propio mito de la caverna de Platón, en donde la actividad científica está marcada al interior de esta por sombras y obstáculos, que establece un único camino que proyecta un final feliz (Medina, 1983).

Siglos después, los epistemólogos, los sociólogos y los historiadores de la ciencia comenzaron su ardua lucha por desmitificar esa ciencia oscura y crear una visión más acorde al trabajo de los científicos y de la propia ciencia. Esta tarea historiográfica que inició a comienzos del siglo XX se denominó internalismo vs. externalismo y se extendió hasta finales de la década de 1980. A lo largo de ese período, las producciones en el ámbito de la historia y la sociología de la ciencia apuntaron a determinar las causas, factores o variables que pudieran explicar los mecanismos de la producción del conocimiento, sus influencias y alcances (Arias y Navarro, 2017).

En este sentido, los estudios sociales de la ciencia inauguraron campos de investigación para comprobar la existencia de las circunstancias sociales, culturales, políticas y económicas que forman parte de la construcción del conocimiento científico en diferentes líneas (Gallego et al., 2009). Algunos sociólogos e historiadores de la ciencia estuvieron interesados en estudiar el ritmo de crecimiento y la dirección de los trabajos científicos, es decir, la formación y actuación de los grupos de científicos tanto institucionalizados como informales. Otros investigadores se centraron en las razones que explican el desarrollo de ciertas clases de investigaciones científicas, las carreras de los científicos y la influencia que esta ha ejercido en la sociedad; y un tercer grupo consideró la constitución social del contenido de las teorías científicas como parte de la historia sociocultural de la humanidad (Merton, 1984; Barona, 1994; Martini, 2008; Shapin 2008).

A partir de esto, y como legado de los estudios sociales de la ciencia, se inició otro conflicto al interior de la ciencia: el reconocimiento y la titularidad de los descubrimientos. Los científicos comenzaron cada vez más moverse entre la academia y las empresas, y búsqueda del reconocimiento reputacional y financiero (Shapin, 2008). Esto es, un enfoque Mertoniano de las recompensas y la concepción de las instituciones de la ciencia como empresas. $Y$ es justo en este momento cuando se sitúa el origen de los estudios de las controversias científicas alrededor de la década de 1970, basados en la sociología del conocimiento científico (Restivo, 1992; Martini, 2011).

\section{Origen de las controversias socio-científi- cas como objeto de estudio}

La sociología de la ciencia y la educación científica han desarrollado diversos estudios sobre las implicaciones de la ciencia en la sociedad (Vessuri, 1992; Restivo, 1992; Vilchez y Furio, 1999; Eslava, 2004; Gallego et al., 2009; López-Cerezo 
y Núñez, 2001; Acevedo et al., 2003; Solbes y Torres, 2015, Acevedo y Carmona, 2016). En estos se ve implicado un enfoque en las cuestiones sociales relacionadas con la ciencia. En este sentido, y desde finales de la década de 1990, se aceptó que la ciencia es un producto de la cultura que la produce y, por tanto, la ciencia es codependiente del contexto social en el que se desarrolla y los compromisos políticos y económicos que la influencian (Bybee, 1997; Fensham, 2002; Hodson, 2003; Lederman, 2007).

A partir de estos planteamientos nace el interés por las cuestiones socio-científicas, relacionadas con problemáticas sociales controvertidas de la ciencia y dilemas socio-éticos (Kolstø, 2001; Ratcliffe y Grace, 2003; Sadler y Zeidler, 2004). El primer tipo de controversia que llamó la atención dentro de la sociología de la ciencia fue la disputa de prioridad. La cual se trata de una confrontación entre uno o más científicos o grupos de científicos que reclaman la autoría de un determinado descubrimiento científico (Pinch, 2015), lo que se convirtió en la primera línea de estudio.

Así, por ejemplo, una de las controversias más nombradas es el caso de la Foto 51, uno de los retos más grandes del siglo XX: la estructura del ADN. Este episodio de la historia de la ciencia comienza con la publicación del artículo "A structure for deoxyribose nucleic acid" en la revista Nature, el 25 de abril de 1953, por Watson y Crick; este artículo significó la culminación del descubrimiento tridimensional de la molécula relacionada con la herencia genética, el ADN. Esta disputa, de tres grupos de investigación en su carrera por hacerse con el descubrimiento, dejó entrever los oscuros intereses de la ciencia. El primer grupo era el liderado por Linus Pualing, en Estados Unidos, quien propuso un primer modelo de triple hélice. Un segundo grupo, liderado por James Dewey Watson y Francis Harry Compton Crick, en Universidad de Cambridge; y, por último, Maurice Wilkins del Kings College de Londres. En la carrera por quedarse con el descubrimiento, y habiendo conocido la aproximación de Pauling, Crick, y Watson, se conocieron los avances de los estudios cristalográficos realizados por Wilkins (Álvarez, 2015).

Fueron Watson y Crick quienes lograron hacerse con la autoría al formular el modelo de la estructura del ADN a partir de la famosa Foto 51 que Rosalind Franklin y Raymond Gosling aplicando la difracción de rayos $X$. En esta foto obtuvieron una imagen nítida la estructura helicoidal del ADN, fotografía que demostró que la estructura era una doble hélice (Álvarez, 2015; Acevedo y Carmona, 2016).

Existen en la literatura muchos otros ejemplos. Uno de estos es el caso de la existencia de una nueva forma de fusión nuclear a temperatura ambiente conocida como "fusión en frío" (Collins, 1992).

Otra perspectiva relacionada con las controversias científicas es la percepción social, relacionada con el impacto negativo de la ciencia y la tecnología en los ciudadanos, lo que ha significado cambios en las políticas públicas y económicas. Tal es el caso de la publicación del libro de Rachel Carson Silent Spring, en el que se denunciaba los efectos nocivos de los pesticidas en la naturaleza, lo que fue el inicio de la preocupación ambiental en el mundo. Estos ejemplos, así como otros más, se han relatado a lo largo de las últimas décadas, lo que termina por demostrar la necesidad de formar a los ciudadanos científicamente y de alertar sobre las diferentes tecnologías utilizadas en la alimentación, la guerra o la industria y sus consecuencias sociales y ambientales.

\section{Las controversias socio-científicas en la educación en Ciencias}

Las controversias socio-científicas han sido definidas desde la educación en ciencias como:

La puesta en práctica en el aula del enfoque CTS y el uso de las controversias socio-científicas como forma de empoderar a los estudiantes como ciudadanos ha resultado útil, como lo afirma Osorio:

Una línea de trabajo académico e investigativo, que tiene por objeto preguntarse por la naturaleza 
social del conocimiento científico - tecnológico y sus incidencias en los diferentes ámbitos económicos, sociales, ambientales y culturales de las sociedades occidentales (principalmente). (2002, p. 3)

De acuerdo con lo anterior, lo que se busca es que las personas logren tener una participación ciudadana en las decisiones tecnológicas que los afecten, a través de una correcta alfabetización tecno-científica que debe ser impartida desde la escuela.

Este enfoque, como lo afirma López (1998), tiene sus orígenes en la década de 1960, a raíz de algunas movilizaciones sociales cuya preocupación era el desarrollo tecnológico, debido a las consecuencias dejadas por la bomba atómica en la Segunda Guerra Mundial y algunos accidentes nucleares. Las reflexiones derivadas de estas movilizaciones lograron abrir un espacio en las academias universitarias enfocadas a la alfabetización científica y tecnológica de la ciudadanía. Vázquez, Acevedo, Manassero y Acevedo (2006) sustentan que tanto la ciencia como la tecnología están relacionadas con diversos tipos de intereses económicos, sociales, políticos, históricos, filosóficos, culturales y ambientales, con lo cual los estudiantes pueden aportar de acuerdo con el contexto el que se encuentran inmersos, teniendo en cuenta, sus intereses, inquietudes y expectativas.

De acuerdo con Quintero (2010), en Colombia, así como en los demás países de América Latina, los estudios sobre las relaciones ciencia, tecnología y sociedad (CTS) están orientados en su mayoría hacia la parte socio-histórica e histórico-filosófico, dejando de lado la investigación. A pesar de la reciente preocupación mundial por las secuelas que ha traído el uso inadecuado de la tecnología, el enfoque CTS se presenta como una renovación al currículo de la educación básica y superior. Sin embrago, la producción académica a nivel investigativo demuestra un interés mínimo hacia la teorización y fundamentación conceptual de este enfoque, razón por la cual se puede concluir que se requiere un arduo trabajo pedagógico para la formación de docentes en ciencias y tecnología en el enfoque CTS. Lo anterior debido a que en las pocas investigaciones encontradas, se evidencia la carencia teórica y didáctica para generar nuevos adelantos en este campo (que impliquen a la formulación de propuestas acordes a las necesidades de formación profesional en países que como el nuestro) se encuentra en un serio atraso científico, tecnológico y social. Por esta misma línea, Solbes, Vilches y Gil (2002) establecen qué se conoce sobre la participación de los docentes de Ciencias en el diseño curricular con enfoque CTS.

Entre las razones por las cuales López y Valenti (1999) plantean que se debe educar para innovar y participar, por lo que esto hace pertinentes las reflexiones, como el diseño de actividades escolares que propicien su logro, respondiendo a la realidad científica y tecnología en el mundo actual y asumiendo valores, estimulando la sensibilidad social y el sentido crítico. Es allí donde la escuela tiene su papel más importante, ya que desde el aula el docente de ciencias y tecnología debe garantizar la formación de los estudiantes en fundamentos técnicos, científicos, históricos, ideológicos y de impacto social y ecológico; así como una racionalidad crítica en el uso y protección de la tecnología. Además, los estudiantes deben implicarse activamente en la organización y desarrollo de los contenidos educativos aportando desde sus experiencias.

Las controversias científicas, por su naturaleza, permiten trabajar en las clases de ciencias diversos enfoques sociales, históricos y políticos. Esto nos podría permitir abarcar contextos cotidianos y problemas actuales, tales como: la educación en energías renovables, los problemas ambientales, los alimentos transgénicos, la democratización del conocimiento, etc. Las miradas que surgen a partir de la conjunción de los estudios sociales y la educación científica, que desde años atrás se ha intentado cambiar y sustituir frente a la fuerte crítica de los procesos de enseñanza aprendizaje que se ejerce en los jóvenes, hacen del enfoque CTS 
uno de los aportes para solventar y acuñar a los nuevos modelos que permitiría consolidar y apoyar dichos procesos educativos (González de la Fe, 2009; Acevedo y Carmona, 2016).

Los estudios de CTS tienen entre sus objetivos la naturaleza social del conocimiento científico-tecnológico y sus incidencias en los diferentes ámbitos económicos, sociales, ambientales y culturales de las sociedades occidentales (principalmente). A los estudios CTS también se les conoce como estudios sociales de la ciencia y la tecnología (Streider et al., 2017). Lo que nos podría enmarcar el estudio de las energías renovables en comunidades indígenas como parte de la formación científica ciudadana desde el compromiso público ascendente, tesis doctoral que se está desarrollando en la Universidad Distrital Francisco José de Caldas (Ballesteros, 2019).

Acudiendo a lo que establece la OEI desde el ámbito educativo, los enfoques CTS suponen la confluencia de propuestas e iniciativas diversas. En este sentido, el uso de controversias socio-científicas podrían permitir trabajar el compromiso público y promover la participación de las comunidades en las decisiones sobre el uso de las energías renovables, el cual es el precedente para la existencia de una ciudadanía con actitudes y capacidades para esa participación democrática. La formación desde esta perspectiva interpela a una población con una concepción más acorde del papel social de la ciencia, lo que implica la necesidad de trabajar el compromiso público ascendente como eje conceptual en el caso de las energías renovables.

Así, desde este enfoque se pueden generar ciertas habilidades y actitudes que propician en los maestros de Ciencias Naturales y estudiantes una concepción científica del mundo, que se encamine hacia un ejercicio de la formación científica, conservación y cuidado de los recursos energéticos, que promueva en los ciudadanos una actitud crítica y que pueda enfrentar los desafíos teniendo en cuenta la actual emergencia planetaria. A partir de esto,
La educación desde el enfoque Ciencia-Tecnología Sociedad intenta promover la alfabetización en ciencia y tecnología para que los ciudadanos sean capaces de participar en el proceso democrático de toma de decisiones y promover la acción ciudadana en la resolución de problemas relacionados con la ciencia y la tecnología. (Membiela y Padilla, 2005, p. 5)

Lo cual nos indica la importancia de propiciar estudios dentro del enfoque. Por su parte, Acevedo afirma lo siguiente:

La tesis fundamental que sustentamos es que el paradigma CTS puede guiar mejor que otros la selección de contenidos básicos, relevantes y más útiles para todos los estudiantes, que se relacionen con la vida cotidiana y puedan contribuir realmente a su alfabetización científica y tecnológica, así como dar pautas metodológicas para llevar a la práctica esta importante innovación educativa. (2016, p. 7)

Siguiendo esta misma línea, la educación desde el enfoque CTS tiene diferentes objetivos, realizables de diversas formas curriculares y extracurriculares, todos relacionados con promover y desarrollar formas de análisis e interpretación sobre la ciencia y la tecnología de carácter interdisciplinario. Entre estos, se destacan la historia, la filosofía y sociología de la ciencia y la tecnología, así como la economía del cambio técnico y las teorías de la educación y del pensamiento político (Osorio, 2002, p. 67).

Desde nuestra perspectiva, el uso de las controversias socio-científicas puede potenciar que los estudiantes logren construir su conocimiento tras el análisis problematizado de las energías renovables dada a la velocidad del cambio tecnológico y las necesidades energéticas que enfrentan las distintas comunidades (Jennings, 2009; Kandpal y Broman, 2014). En este sentido, Ballesteros propone en 2019 trabajar la educación en energías renovables desde las siguientes premisas: 
1. Las fuentes no convencionales de energía renovable con un énfasis particular en algunas específicas dependiendo de las necesidades locales y la disponibilidad de recursos.

2. Los aspectos pertinentes para el desarrollo y la difusión de soluciones basadas en energías renovables, tales como: 1) evaluación de recursos; 2) diseño, fabricación, instalación, monitoreo del rendimiento, resolución de problemas y mantenimiento de tecnologías; 3) finanzas, aspectos económicos y energéticos de la utilización de la tecnología de energía renovable; 4) aceptabilidad sociocultural; y 5) evaluación de los impactos ambientales asociados.

3. La conservación de la energía y la interacción entre la energía y el medio ambiente.

4. El equilibrio entre la teoría y la práctica. Por ello, sus contenidos deben incluir aportes sobre experimentos de laboratorio y demostración, capacitación práctica, resolución de problemas, diseño y fabricación de aportes; además de conferencias, tutoriales, tareas y seminarios, etc.

5. Facilitar el intercambio de experiencias energéticas efectivas y mutuamente beneficiosas.

6. El emprendimiento de manera que los estudiantes puedan acercarse a distintas opciones del mercado laboral y la implentación de soluciones de Investigación y Desarrollo I+D.

Se debe proporcionar preferiblemente en los idiomas locales para una mayor aceptación, asegurando que los materiales y recursos de enseñanza y aprendizaje deben ser accesibles y de buena calidad. Ya que las controversias no solo hacen referencia a episodios históricos de la ciencia, también se utilizan hoy día para que los estudiantes actúen no solo como científicos, sino como ciudadanos científicamente alfabetizados que toman partido de una situación desde su conocimiento y experiencias cotidianas. En este caso, el papel de los procesos en este campo de acción debe ser el menos visible, destacándose más por ser un director de investigación suscitando preguntas, nunca respuestas.

En este modelo, los estudiantes deben realizar un proceso de construcción social de teorías y enfoques a través de la generación y resolución de problemas teóricos y prácticos. Por tanto, el currículo para este caso está centrado en la resolución de problemas desde el análisis del conocimiento disciplinar incluyendo, a diferencia de los demás, la historia de la ciencia, pues se asume que el aprendizaje de los estudiantes debe asemejarse con el mismo proceso de construcción de los contenidos científicos.

Las actividades de enseñanza que elabora el profesor deben incluir los siguientes aspectos: un motivador, definición de un problema e identificación de variables, emisión de hipótesis, posibles estrategias de resolución. Explicación fundamentada de la estrategia, un análisis de resultados de acuerdo con las hipótesis hechas anteriormente, reflexión del proceso realizado y elaboración de una memoria que evidencie el análisis del proceso y de los resultados.

Este enfoque exige que el profesor cuente con un gran dominio de sus conocimientos disciplinares, así como también desde la parte pedagógica, ya que es el encargado de realizar los ajustes pertinentes para que los estudiantes encaminen, enriquezcan o redefinan sus investigaciones.

De acuerdo con los enfoques anteriores, Gónzalez y Orribo (1995) argumentan la posibilidad de enseñar de distintas formas en la clase de Física, para lo cual se establecen varios enfoques didácticos. Ellos concluyen que el comportamiento de los docentes de esta área se adapta a cinco enfoques didácticos muy caracterizados, como lo son: transmisor, tecnológico, artesano, descubridor y constructivista. Por otro lado, Boyer y Tiberghien (1989) revelan en su artículo las representaciones complejas y a veces ambivalentes de la enseñanza de las ciencias (Física y Química), por parte de los docentes y de los estudiantes, además de establecer las finalidades de estas ciencias según el punto de vista de cada actor del 
aula de clase, hace un recorrido por la problemática y enfoques de investigación concluyendo que se necesita una urgente profundización de la reflexión sobre lo que se busca en la enseñanza de la Física en el aula.

Estas razones permiten entrever que a través de la utilización del enfoque CTS en el currículo de la enseñanza de las ciencias los docentes se dan a la tarea de formar estudiantes críticos en el uso de la tecnología; estimulando la creatividad y el diseño siempre en pro de responder a problemas sociales mejorando su entorno, acentuando su participación social a través de la innovación dando un sentido y uso a su conocimiento y fundamentación técnica y científica, asumiendo la tecnología como lo plantean Vaccarezza et al.:

[...] el conjunto de saberes inherentes al diseño y concepción de los instrumentos (artefactos, sistemas, procesos y ambientes) creados por el hombre a través de su historia para satisfacer sus necesidades y requerimientos personales y colectivos. (1998, p.114)

Dado que el desarrollo del país depende del conocimiento y la necesidad de actuar sobre la emergencia de las energías renovables, se debe forjar una sociedad pensante, argumentativa, con cultura ecológica y propiciar la participación ciudadana en situaciones contextualizadas y focalizadas.

\section{A manera de conclusión: las controversias científicas y su papel en la educación en ciencias}

La importancia de incluir en las clases de Ciencias diferentes casos históricos y sociales alrededor de la construcción social del conocimiento científico, han sido utilizados para trabajar la alfabetización científica, las actitudes hacia la ciencia, el desarroIlo del pensamiento crítico, la formación científica ciudadana, entre otros.

La resolución de dichos problemas no es simple ni directa, y la información científica disponible suele ser contradictoria y provenir de ciencia de frontera. La definición de las controversias científicas, tal y como lo expresan Moreno y Jiménez-Liso en su artículo:

Son aquellas disyuntivas sociales que surgen $y$ que están relacionadas con la ciencia, debido a la compleja relación que existe entre ciencia y sociedad. Si solamente pertenecieran al campo de lo social no serían controversias socio-científicas. Aparece la controversia cuando existe diferencia de opiniones relacionadas con estos asuntos, normalmente entre periodistas, ciudadanos y científicos. (2012, p.55)

Esta diferencia de opiniones es debida a que generalmente estas controversias están permeadas por la parte ambiental, ética, económica y hasta religiosa, ayudando en el estudiante a estimular y fortalecer la comprensión del mundo social en el que viven, con aplicación real de los conocimientos adquiridos en sus clases de física (García y Martínez, 2015). Todo esto con el fin de contribuir en la educación para la sostenibilidad, pues las CSC trata de problemas abiertos, complejos y controvertidos, muchos de ellos sin respuestas definitivas, y cualquiera que sea la postura que el individuo o la sociedad tenga ante ellos el debate no le va a ser ajeno, ya que la importancia del mismo va a ir en aumento a medida que prosiguen los avances de la ciencia y los problemas ambientales (España y Prieto, 2009).

Se ha argumentado que, para empoderar a los estudiantes como ciudadanos, es necesario hacer hincapié en la ciencia como institución y los procesos por los cuales se produce el conocimiento científico.

Las consecuencias de las evaluaciones de riesgo y de la toma de decisiones colectivas a menudo tienen consecuencias de gran alcance, haciendo primordial una interpretación adecuada de las afirmaciones de conocimiento relacionadas con las ciencias involucradas. 


\section{Referencias}

Abd-El-Khalick, F.; Lederman, N. G. (2000). The influence of history of science courses on students' views of nature of science. Journal of Research in Science Teaching: The Official Journal of the National Association for Research in Science Teaching, 37(10), 1057-1095. https:// doi.org/10.1002/1098-2736(200012)37:10\%3C1057::AID-TEA3\%3E3.0.CO;2-C

Acevedo-Díaz, J. A.; García-Carmona, A. (2016). Rosalind Franklin y la Estructura Molecular del ADN: un caso de historia de la ciencia para aprender sobre la naturaleza de la ciencia. Revista Científica, 2(25), 162175. https://doi.org/10.14483/udistrital.jour. $\underline{\mathrm{RC} .2016 .25 . \mathrm{a} 2}$

Acevedo-Díaz, J. A.; del Mar Aragón-Méndez, M.; García-Carmona, A. (2018). Comprensión de futuros profesores de ciencia sobre aspectos epistémicos de la naturaleza de la ciencia en cuatro controversias de historia de la ciencia. Revista Científica, 3(33), 344-355. https:// doi.org/10.14483/23448350.13355

Acevedo-Diaz, J. A.; Vázquez, A.; Manassero, M. (2003). Papel de la educación CTS en una alfabetización científica y tecnológica para todas las personas. Revista Electrónica de Enseñanza de las Ciencias, 2(2), 80-111.

Álvarez, J. P. (2015). Rosalind Franklin y el descubrimiento de la estructura del ADN. Revista Médica Clínica Las Condes, 26(4), 544-549. https://doi.org/10.1016/j.rmclc.2015.07.007

Aragón-Méndez, M.; García-Carmona, A.; Acevedo-Díaz, J. A. (2016). Aprendizaje de estudiantes de secundaria sobre la naturaleza de la ciencia mediante el caso histórico de semmelweis y la fiebre puerperal. Revista Científica, 4(27), 302-317. https://doi.org/10.14483/ udistrital.jour.RC.2016.27.a1

Arias-Monge, M.; Navarro-Camacho, M. (2017). Epistemology, science and scientific education: premises, questions and reflections to think science culture. Actualidades Investigativas en Educación, 17(3), 774-794. https://doi. org/10.15517/aie.v17i3.29878

Barona, J. L. (1994). Ciencia e historia. En Debates y tendencias en la historiografía de la ciencia. Valencia: Guada.

Ballesteros-Ballesteros, V. A. (2019). La educación en energías renovables como alternativa de promoción del compromiso público ascendente entre los indígenas Wayuu en la Alta Guajira. Revista Científica, 388-397.

Bybee, R. (1997). Towards an Understanding of Scientific Literacy. En W. Graeber y C. Bolte (eds.), Scientific Literacy. Kiel: IPN.

Eslava, J. C. (2004). Tensiones y confluencias: Una mirada fugaz al triple legado de los estudios histórico-sociales sobre la ciencia. Revista Colombiana de Sociología, 23, 159-180.

Fensham, P. J. (2002): Time to Change Drivers for Scientific c Literacy. Canadian Journal of Science, Mathematics and Technology Education, 2(1), 9-24. https://doi. org/10.1080/14926150209556494

Fouréz, G. (1994). Alfabetización científica y tecnológica. Buenos Aires: Calihue.

Gallego-Torres, P.; Zapata, J.; Rueda, M. (2009). Una alfabetización científica tecnológica y cultural. Revista Científica, 11, 52-61.

González de la Fe, T. (2009). El modelo de triple hélice de relaciones universidad, industria y gobierno: un análisis crítico. Arbor, 185(738), 739-755. https://doi.org/10.3989/ arbor.2009.738n1049

Hodson, D. (2008). Towards scientific literacy. A teachers' guide to the history, philosophy and sociology of science. Rotterdam: Sense.

Jennings, P. (2009). New directions in renewable energy education. Renewable Energy, 34(2), 435-439. https://doi.org/10.1016/j. renene.2008.05.005

Kelly, G. J.; Carlsen, W.S.; Cunningham, C. M. (1993). Science education in sociocultural context: Perspectives from the sociology of science. Science Education, 77(2), 207-220. https://doi.org/10.1002/sce.3730770208 
Kandpal, T. C.; Broman, L. (2014). Renewable energy education: A global status review. Renewable and Sustainable Energy Reviews, 34, 300-324. https://doi.org/10.1016/j. rser.2014.02.039

Lederman, N. (2007). Nature of science, past, present and future. En A. Abel y N. Lederman (eds.), Handbook of Research in Science Education (pp. 831-879). Mahwah: Erlbaum

López-Cerezo, J. A.; Núñez, J. (2001). Innovación tecnológica, innovación social y estudios CTS en Cuba. En Desafíos y tensiones actuales en ciencia, tecnología y sociedad (pp. 289-308). Biblioteca Nueva.

Martini, M. (2011). La relación Merton-Shapin a partir del debate historiográfico internismo/externismo. Cinta de Moebio, 42, 288-301.

Martini, M. (2008). Reflexiones acerca del debate historiográfico internalismo/externalismo en la obra de Steven Shapin. En H. Fass y H. Severgnini (eds.), Epistemología e Historia de la Ciencia (pp. 317-323). Córdoba: Universidad Nacional de Córdoba.

Merton, R. K. (1984). Ciencia, tecnología y sociedad en la Inglaterra del siglo XVII. Madrid: Alianza.

Moreno, N. D. (2019). Caracterizando controversias socio científicas en la prensa. Una herramienta para el desarrollo de la alfabetización científica. Revista Eureka sobre Enseñanza y
Divulgación de las Ciencias, 16(1), 1102-1. https://doi.org/10.25267/Rev Eureka ensen divulg_cienc.2019.v16.i1.1102

Pinch, T. (2015). Scientific controversies. En, International Encyclopedia of the Social \& Behavioral Sciences, 2nd edition, vol. 21. https://doi. org/10.1016/B978-0-08-097086-8.85043-6

Restivo, S. (1992). La ciencia moderna como problema social. Fin de Siglo, 3, 20-39.

Solbes-Matarredona, J.; Torres-Merchán, N. Y. (2015). Alternativas para reflexionar aspectos críticos de la ciencia en el aula-Alternatives to Reflect on Critical Aspects of Science in the Classroom. Revista Científica, 2(22), 31-44. https://doi.org/10.14483/10.14483/udistrital. jour.RC.2015.22.a3

Torres-Merchán, N.; Solbes, J. (2016). Contribuciones de una intervención didáctica usando cuestiones socio científicas para desarrollar el pensamiento crítico. Enseñanza de las Ciencias: Revista de Investigación y Experiencias Didácticas, 34(2), 43-65. https://doi.org/10.5565/ rev/ensciencias. 1638

Vessuri, H. M. C. (1992). Perspectivas recientes en el estudio social de las ciencias. Fin de Siglo, 3, 40-52.

Vilches, A.; Furió, C. (1999). Ciencia, tecnología, sociedad: implicaciones en la educación científica para el siglo XXI. Biblioteca Digital da OEl. 